\title{
Data assimilation approaches in the EURANOS project
}

\author{
J.C. KAISER ${ }^{1}$, F. GERING ${ }^{2}$, P. ASTRUP ${ }^{3}$, T. MIKKELSEN ${ }^{3}$
}

\begin{abstract}
Within the EURANOS project data assimilation (DA) approaches have been successfully applied in two areas to improve the predictive power of simulation models used in the RODOS and ARGOS decision support systems. For the areas of atmospheric dispersion modelling and of modelling the fate of radio-nuclides in urban areas the results of demonstration exercises are presented here. With the data assimilation module of the RIMPUFF dispersion code, predictions of the gamma dose rate are corrected with simulated readings of fixed detector stations. Using the DA capabilities of the IAMM package for mapping the radioactive contamination in inhabited areas, predictions of a large scale deposition model have been combined with hypothetical measurements on a local scale. In both examples the accuracy of the model predictions has been improved and the uncertainties have been reduced.
\end{abstract}

\section{Introduction}

After nuclear emergencies the assessment of the current state of radioactive contamination and the prediction of the future situation are crucial information for decision makers. Measurements and model predictions are the main sources of raw data after an accident. In many cases measurements are more accurate and less uncertain than model results. However, decision making cannot be based entirely on measurements mainly for two reasons. Firstly, measurements do not possess predictive power and secondly, especially in the early phase of an accident, there may not be enough of them. Very often an improved picture of the situation can be obtained by combining measurements and model predictions in a Bayesian approach referred to as data assimilation. In the EURANOS project data assimilation techniques have been applied in two areas: modelling of atmospheric dispersion and modelling the radioactive contamination in urban areas. For these areas demonstration cases are presented in this article. The mathematical algorithms for data assimilation are based on different versions of the Kalman filter (Kalman, 1960). Basic equations are given in the Appendix.

\footnotetext{
Helmholtz-Zentrum München - Institute of Radiation Protection, Ingolstädter Landstr. 1, 85764 Neuherberg, Germany.

Bundesamt für Strahlenschutz - SW 3.2, Ingolstädter Landstr. 1, 85764 Oberschleißheim, Germany.

Risø National Laboratory DTU, PO Box 49, 4000 Roskilde, Denmark.
} 


\section{Data assimilation for atmospheric dispersion}

Before the start of the EURANOS project a data assimilation module has been developed for the RIMPUFF atmospheric dispersion code (Astrup et al., 2004). Within the project this module has been decoupled from RIMPUFF and made available for implementation with other atmospheric dispersion codes.

A Kalman filter operates on a so called state vector, a series of variables predicted by the code and influencing the results to be compared with the measurements. In the case of RIMPUFF the state vector contains for each puff its position and total inventory, i.e. sum over the activity concentrations for all nuclides. Puff positions and inventories are determining the gamma dose rates at ground level. Hence, the measurements used for the Kalman filtering are gamma dose rates at ground level detector points averaged over 10 minutes.

\section{Test cases}

With the lack of real measured accident data, tests of the RIMPUFF integrated Kalman filter have had to be carried out using simulated measurements. One way is to use the RIMPUFF code itself to generate "measurement data". Then in the Kalman test run one or more parameters influencing the gamma dose rate at the selected detector points are perturbed. Perturbations can be assigned to the released inventory, wind speed, wind direction, height of release etc.

For the reported cases the basic "measurement data" generating run has a release point at Risø, Denmark, a constant wind from west, and the gamma dose rates are collected at 8 detector points surrounding Ris $\varnothing$ at $2 \mathrm{~km}$ distance plus at 15 detector points arranged as three rows of five points. The central row stretches directly east from Ris $\varnothing$ at 10 to $50 \mathrm{~km}$ distance, the other two rows are located parallel, running $10 \mathrm{~km}$ north and south of the central row (see Fig. 1).

Case 1: release halved and doubled relative to that of the "measurement" generating run, Kalman filtering only on 8 detector points which surround the release point. Results are shown in Figure 2 for the point $50 \mathrm{~km}$ exactly downstream, i.e. below the centre of the plume. It is seen that with a reasonable large anticipated release uncertainty, characterised by the parameter $a$, the Kalman filter catches very well the "measurements" and moves the calculation results in line with these monitoring data.

Case 2: basic release but wind 5 degree off the western direction, Kalman filtering based on measurements in the 8 points surrounding the release point plus in (a) the 3 easternmost points of the $3 \times 5$ detector point array, see Figure 3 ; and (b) all 15 points, see Figure 4. 


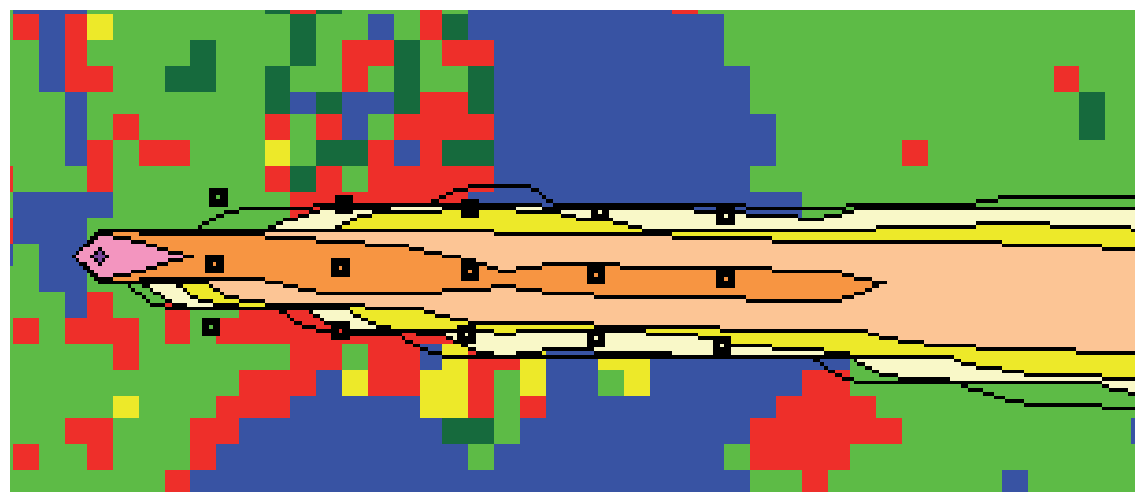

Figure 1 - Detector point layout, black squares. The 8 points surrounding the release point are not shown. Contours: Gamma doses [Gy] from "measurement” generating run.

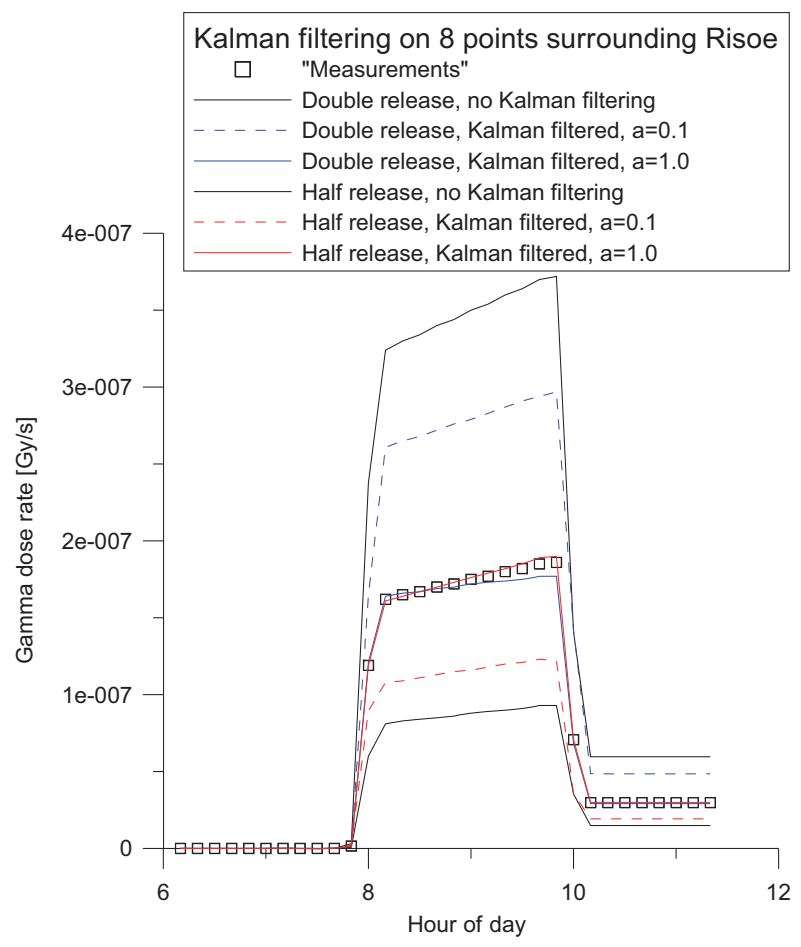

Figure 2 - Gamma dose rates $50 \mathrm{~km}$ from the release point, right below the plume. Kalman filtering applied in runs with the release doubled and halved relative to the "measurement"-generating run, and with different release rate uncertainties " $a$ ". 

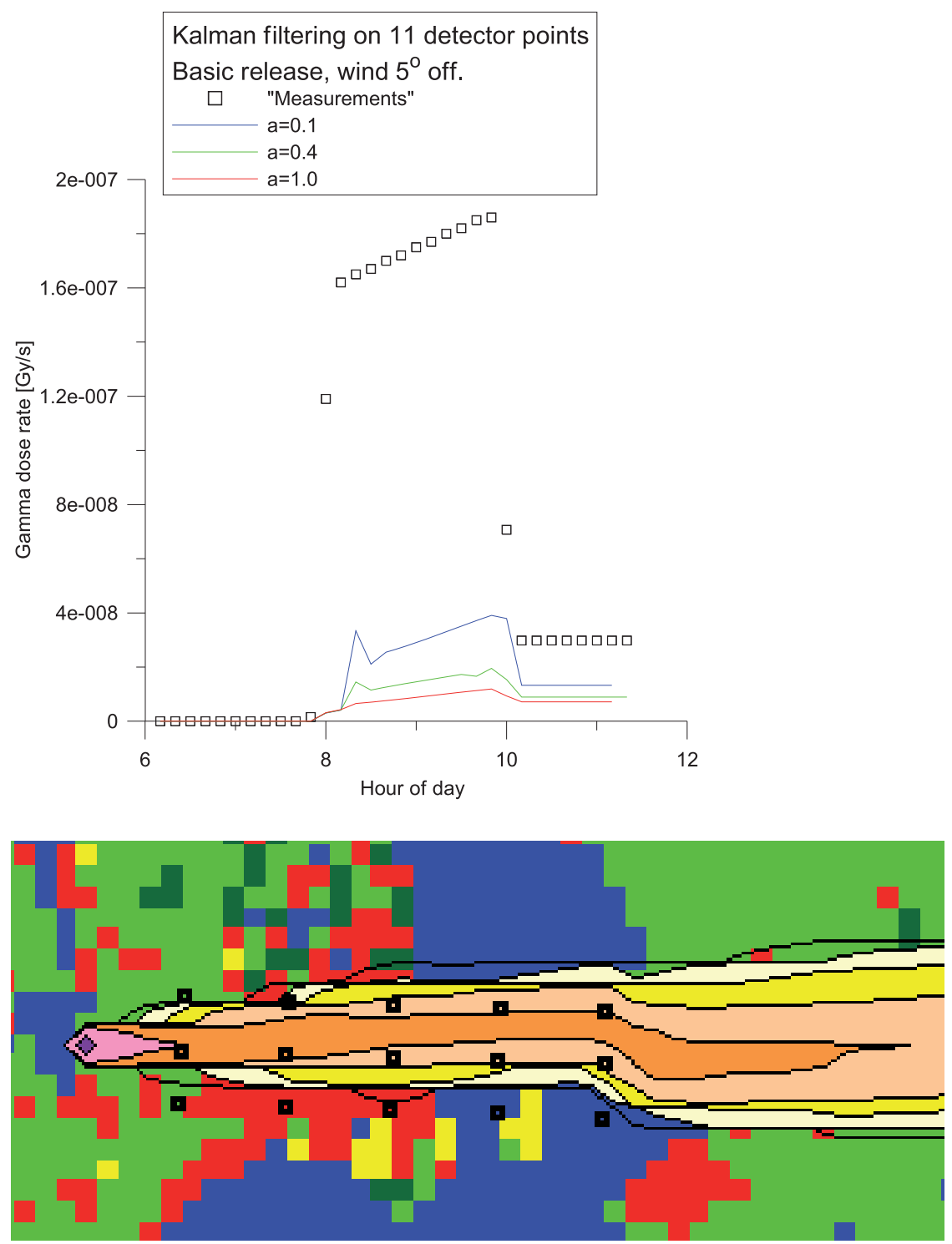

Figure 3 - Gamma dose rate fields with wind $5^{\circ}$ south of west. Kalman filtering on the 8 detector points surrounding the release point plus the 3 eastern most detector points in the $3 * 5$ array. Relative release uncertainty: $a=0.1$. 

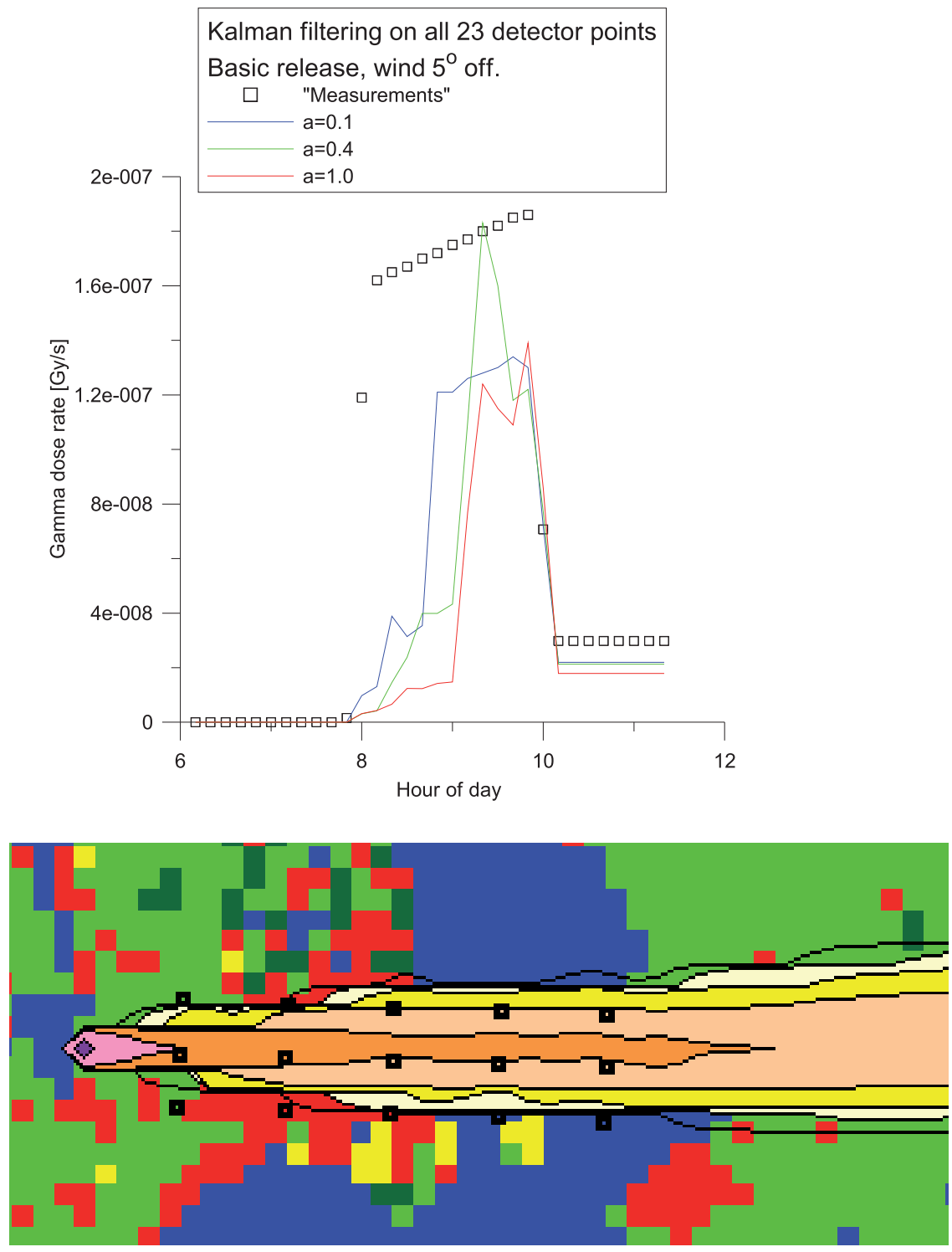

Figure 4 - Gamma dose rate fields with wind $5^{\circ}$ south of west. Kalman filtering on the 8 detector points surrounding the release point plus all 15 array detector points. Relative release uncertainty: $a=0.1$. 
As a general observation we may state that the correction is performed better in case more detector stations are triggered by the plume radiation.

\section{Data assimilation in urban areas}

After deposition of radioactive aerosols in urban areas inhabitants may be exposed to radiation and an estimation of the external dose is very important. To estimate the exposure, maps of the surface concentration can be applied as a starting point. Inhomogeneities from buildings, roads and various other forms of land will cause uncertainties which cannot be accounted for in detail in these maps. Nevertheless, efforts should be made to reduce them as much as possible. Hence, the EURANOS consortium commissioned the development of the Inhabited Areas Monitoring Module (IAMM) for this task. IAMM, which has already been integrated into RODOS and ARGOS, operates in two modes (Kaiser and Pröhl, 2007) either (a) based alone on measurements of the external gamma dose rate using geostatistical interpolation or (b) in combination with model predictions using data assimilation. In a hypothetical scenario for the detonation of a radioactive dispersion device IAMM has been successfully applied with geo-statistical interpolation (Thiessen et al., 2009).

In this article the data assimilation mode is demonstrated with simulated data for a hypothetical accident of a nuclear reactor located in Karlsruhe (Germany). Model predictions for surface contamination of a reference area of infinitely extended lawn were generated with an ALSMC run of RODOS with an inventory of $10 \%{ }^{134} \mathrm{Cs}$ and $90 \%{ }^{137} \mathrm{Cs}$ (Fig. 5, left panel). During the release a wind speed of $5 \mathrm{~m} / \mathrm{s}$ in eastern direction was assumed. The right panel of Figure 5 depicts a crop-out of an inhabited area, encircled by a black square in the left panel of Figure 5, with 16 cells of length $4 \mathrm{~km}$.

For this area of interest 32 hypothetical measurements of the gamma dose rate were generated, which are caused by a reference surface contamination shown in the left panel of Figure 6. To each measurement a lognormal uncertainty distribution has been assigned. The arithmetic standard deviation has been set by a factor of 10 larger than the actual measurement value.

Using an Ensemble Kalman Filter (EnKF) (Evensen, 2003; Gering, 2005) measurements (Fig. 6, left) and model predictions (Fig. 5, right) were combined on a grid of $100 \mathrm{~m}$ cell length. In the EnKF, the error covariance matrices are represented by ensembles of probable values. From experience it is known, that 100 ensemble members are sufficient (Gering, 2005). The state vector consists of 1600 grid values of the reference surface contamination. Their uncertainties are represented by 100 realisations for each grid point provided by the simulation model. 


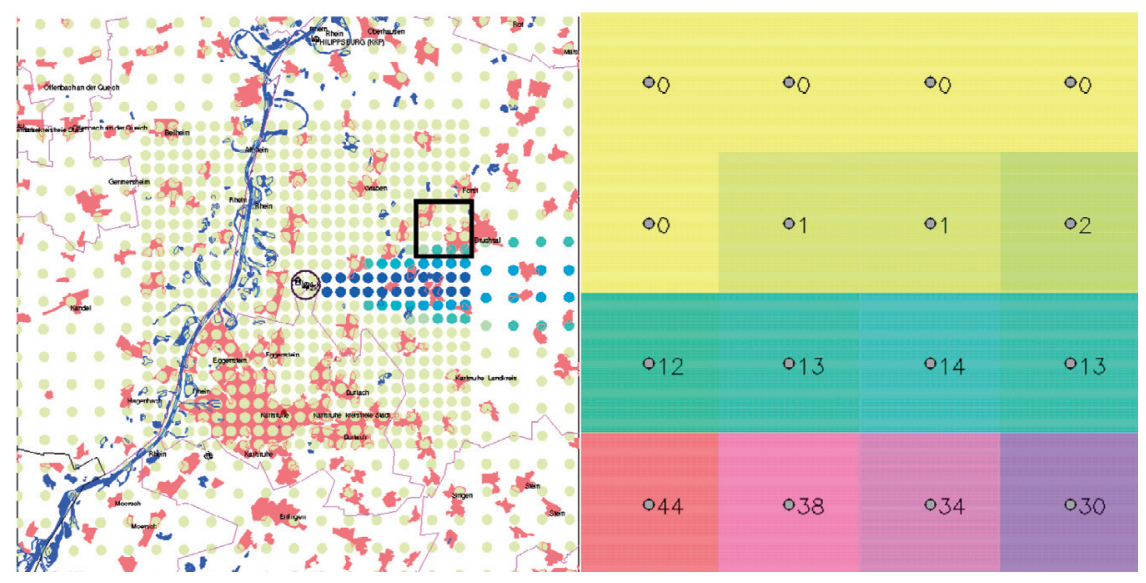

Figure 5 - Model prediction for the surface contamination $\left[\mathrm{kBq} / \mathrm{m}^{2}\right]$ from a run of RODOS module ALSMC, left: for a large area of $168 \times 168 \mathrm{~km}$, right crop-out of an urban area (encircled by a black square in the left panel) with 16 cells of length $4 \mathrm{~km}$.

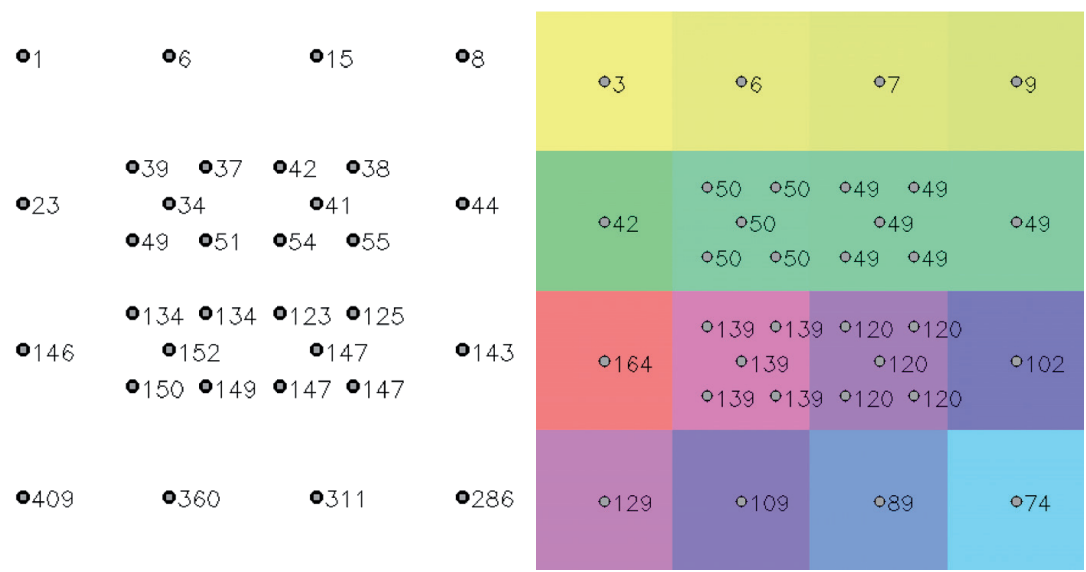

Figure 6-Hypothetical measurements (left) of the reference surface contamination $\left[\mathrm{kBq} / \mathrm{m}^{2}\right]$ in the cropout and result of data assimilation (right).

By comparing Figures 5 and 6 one notes that following the data assimilation process, the general pattern of the model prediction is preserved. The prediction provides the backbone for the resulting map but the individual grid values are drawn to the measurement values, especially if spatial density of the measurements is large. 


\section{Concluding remarks}

For highly dynamic atmospheric dispersion applications data assimilation algorithms can always display their potential for improvement if the density of detector points is sufficiently high and the wind is reasonably well defined. However, in urban areas data assimilation should be applied only in the early phase after cloud deposition when only a few measurements are available. In the later phase enough measurements can be expected to rely fully on interpolation methods. Both demonstration exercises showed that the accuracy of a model prediction is improved and its uncertainty is reduced if a prediction is combined with measurements via data assimilation.

\section{Appendix: basic kalman filter equations}

The Kalman filtering process is described as series of matrix equations repeatedly applied at every time step:

$\bar{x}=M \hat{x}+h$

$P=M P M^{T}+Q$

$\bar{y}=H \bar{x}+g$

$K=\bar{P} H^{T}\left(H \bar{P} H^{T}+R\right)^{-1}$

$\hat{x}=\bar{x}=K(\hat{y}-\bar{y})$

$P=(I-K H) \bar{P}(I-K H)^{T}+K R K^{T}$ prediction update of state vector;

prediction update of error covariance matrix; prediction of the data to be measured; calculation of Kalman gain;

Kalman filtering;

final update of error covariance matrix.

The first equation is a linearization of the model prediction (e.g. from RIMPUFF for atmospheric dispersion or from ALSMC for urban areas). It updates the state vector $x$ in time. In the second equation $Q$ denotes the uncertainty matrix for this update; $P$ is the error covariance matrix. The third equation is a linearization of the measurement data prediction, $K$ is called the Kalman gain, $y$ is the measurement vector, and $R$ is the measurement uncertainty.

Note, that the error covariance matrix $R$ needs to be known before the first time step, i.e. to be given initial values. For a normal well behaved Kalman filter with a constant set of state vector variables $R$ will stabilize with time and the initialization is not critical. In the RIMPUFF application the amount of state vector variables change with every new puff and with every puff leaving the calculation area and the initial values set for $R$ are of high importance.

Acknowledgment. This work has received partial financial support from the European Commission Sixth Framework Programme (Nuclear Fission/Radiation 
Protection) under the EURANOS integrated project: European approach to nuclear and radiological emergency management and rehabilitation strategies (Contract No: FI6R-CT-2004-508843).

\section{REFERENCES}

Astrup P., Turcanu C., Puch R.O., Rojas-Palma C., Mikkelsen T. (2004) Data assimilation in the early phase: Kalman filtering RIMPUFF, Ris $\varnothing-\mathrm{R}-1466(\mathrm{EN})$, www.risoe.dk/rispubl/VEA/ris-r1466.htm.

Evensen G. (2003) The Ensemble Kalman Filter: Theoretical Formulation and Practical Implementation, Ocean Dyn. 53, 343-367.

Gering F. (2005) Data assimilation methods for improving the prognoses of radionuclide deposition from radio-ecological models with measurements, $\mathrm{Ph}$.D. Thesis, Leopold-Franzens-Universität Innsbruck, Austria, 145 p.

Kaiser J.C., Pröhl G. (2007) Harnessing monitoring measurements in urban environments for decision making after nuclear accidents, Kerntechnik 7, 218-221.

Kalman R.E. (1960) A new approach to linear filtering and prediction problems, Trans. ASME. J. Basic Eng. 82, 35-45.

Thiessen K.M., Andersson K.G., Batandjieva B., Cheng J.-J., Hwang W.-T., Kaiser J.C., Kamboj S., Steiner M., Tomas J., Trifunovic D., Yu C. (2009) Modelling the long-term consequences of a hypothetical dispersal of radioactivity in an urban area including remediation alternatives, J. Environ. Radioact. 100, 445-455. 